This postprint is published in:

Journal de Mathématiques Pures et Appliquées, Volume 136, 2020, 1-21

DOI: 10.1016/j.matpur.2020.02.004

\title{
POSITIVE SOLUTIONS FOR NONLINEAR NEUMANN PROBLEMS WITH SINGULAR TERMS AND CONVECTION
}

\author{
NIKOLAOS S. PAPAGEORGIOU, VICENŢIU D. RĂDULESCU, AND DUŠAN D. REPOVŠ
}

\begin{abstract}
We consider a nonlinear Neumann problem driven by the $p$-Laplacian. In the reaction term we have the competing effects of a singular and a convection term. Using a topological approach based on the Leray-Schauder alternative principle together with suitable truncation and comparison techniques, we show that the problem has positive smooth solutions.
\end{abstract}

\section{INTRODUCTION}

Let $\Omega \subseteq \mathbb{R}^{N}$ be a bounded domain with a $C^{2}$-boundary $\partial \Omega$. In this paper, we study the following nonlinear Neumann problem with singular and convection terms

$$
\left\{\begin{array}{l}
-\Delta_{p} u(z)+\xi(z) u(z)^{p-1}=u(z)^{-\gamma}+f(z, u(z), D u(z)) \text { in } \Omega, \\
\frac{\partial u}{\partial n}=0 \text { on } \partial \Omega, u>0,1<p<\infty, 0<\gamma<1 .
\end{array}\right\}
$$

In this problem, $\Delta_{p}$ denotes the $p$-Laplacian differential operator defined by

$$
\Delta_{p} u=\operatorname{div}\left(|D u|^{p-2} D u\right) \text { for all } u \in W^{1, p}(\Omega), 1<p<\infty .
$$

In the reaction term (the right-hand side) of the problem, we have the competing effects of the singular term $u^{-\gamma}$ and the convection term $f(z, x, y)$ (that is, the perturbation $f$ depends also on the gradient $D u$ ). The function $f(z, x, y)$ is Carathéodory (that is, for all $(x, y) \in \mathbb{R} \times \mathbb{R}^{N}$ the mapping $z \mapsto f(z, x, y)$ is measurable, and for almost all $z \in \Omega$ the mapping $(x, y) \mapsto f(z, x, y)$ is continuous).

The key feature of this paper is that we do not impose any global growth conditions on the function $f(z, \cdot, y)$. Instead, we assume that $f(z, \cdot, y)$ exhibits a kind of oscillatory behavior near zero. In this way we can employ truncation techniques and avoid any growth condition at $+\infty$. In the boundary condition, $\frac{\partial u}{\partial n}$ denotes the normal derivative of $u$, with $n(\cdot)$ being the outward unit normal on $\partial \Omega$.

The presence of the gradient $D u$ in the perturbation $f$, excludes from consideration a variational approach to dealing with (1). Instead, our main tool is topological and is based on the fixed point theory, in particular, on the Leray-Schauder principle (see Section 2).

Equations with singular terms and equations with convection terms have been investigated separately, primarily in the context of Dirichlet problems. For singular problems, we mention the works of Giacomoni, Schindler \& Takac [7], Hirano, Saccon \& Shioji [1], Papageorgiou \& Rădulescu [16], Papageorgiou, Rădulescu \& Repovš [19, 21], Papageorgiou \& Smyrlis [22, 23], Perera \& Zhang [24], and $\mathrm{Su}, \mathrm{Wu} \&$ Long [27]. For problems with convection, we mention the works of de Figueiredo, Girardi \& Matzeu [1], Gasinski \& Papageorgiou [5], Girardi \& Matzeu [8], Huy, Quan \& Khanh [13], Papageorgiou, Rădulescu \& Repovš [20], and Ruiz [26]. Of the aformentioned works, only Gasinski \& Papageorgiou [5] and Papageorgiou, Rădulescu \& Repovš [20] go outside the Dirichlet framework and deal with Neumann problems. A good treatment of semilinear parametric elliptic equations with both singular and convection terms and Dirichlet boundary condition can be found in Ghergu \& Rădulescu [6, Chapter 9].

Key words and phrases. Singular term, convection term, nonlinear regularity, nonlinear maximum principle, LeraySchauder alternative theorem, fixed point theory

2010 AMS Subject Classification: 35B50, 35J75, 35J92, 35P30, 47H10, 58J20. 


\section{Mathematical BaCKGRound AND hypotheses}

As we have already mentioned, our method of proof is topological and is based on the fixed point theory, in particular, on the Leray-Schauder alternative principle.

Let $V, Y$ be Banach spaces and $g: V \rightarrow Y$ a map. We say that $g(\cdot)$ is "compact" if $g(\cdot)$ is continuous and maps bounded sets of $V$ into relatively compact subsets of $Y$.

We now recall the Leray-Schauder alternative principle (see, for example, Gasinski \& Papageorgiou [2, p. 827] or Granas \& Dugundji [9, p. 124]).

Theorem 2.1. If $X$ is a Banach space and $g: X \rightarrow X$ is compact, then one of the following two statements is true:

(a) $g(\cdot)$ has a fixed point;

(b) the set $K(g)=\{u \in X: u=\operatorname{tg}(u), 0<t<1\}$ is unbounded.

In what follows, we denote by $\langle\cdot, \cdot\rangle$ the duality brackets for the pair $\left(W^{1, p}(\Omega)^{*}, W^{1, p}(\Omega)\right)$ and by $\|\cdot\|$ the norm on $W^{1, p}(\Omega)$. Hence

$$
\|u\|=\left(\|u\|_{p}^{p}+\|D u\|_{p}^{p}\right)^{1 / p} \text { for all } u \in W^{1, p}(\Omega) .
$$

In the analysis of problem (1), we will make use of the Banach space $C^{1}(\bar{\Omega})$. This is an ordered Banach space with positive (order) cone

$$
C_{+}=\left\{u \in C^{1}(\bar{\Omega}): u(z) \geqslant 0 \text { for all } z \in \bar{\Omega}\right\} .
$$

This cone has a nonempty interior which is given by

$$
D_{+}=\left\{u \in C_{+}: u(z)>0 \text { for all } z \in \bar{\Omega}\right\} .
$$

In fact, $D_{+}$is also the interior of $C_{+}$when the latter is furnished with the relative $C(\bar{\Omega})$-norm topology.

Let $A: W^{1, p}(\Omega) \rightarrow W^{1, p}(\Omega)^{*}$ be the nonlinear operator defined by

$$
\langle A(u), h\rangle=\int_{\Omega}|D u|^{p-2}(D u, D h)_{\mathbb{R}^{N}} d z \text { for all } u, h \in W^{1, p}(\Omega) .
$$

The next proposition summarizes the main properties of this operator (see Motreanu, Motreanu \& Papageorgiou [15, p. 40]).

Proposition 2.2. The operator $A: W^{1, p}(\Omega) \rightarrow W^{1, p}(\Omega)^{*}$ is bounded (that is, A maps bounded sets to bounded sets), continuous, monotone (hence also maximal monotone) and of type $(S)_{+}$, that is,

$$
u_{n} \stackrel{w}{\rightarrow} u \text { in } W^{1, p}(\Omega) \text { and } \limsup _{n \rightarrow \infty}\left\langle A\left(u_{n}\right), u_{n}-u\right\rangle \leqslant 0 \Rightarrow u_{n} \rightarrow u \text { in } W^{1, p}(\Omega) .
$$

For the potential function $\xi(\cdot)$, we assume the following:

$H(\xi): \xi \in L^{\infty}(\Omega), \xi(z) \geqslant 0$ for almost all $z \in \Omega, \xi \not \equiv 0$.

The following lemma will be helpful in producing estimates in our proofs.

Lemma 2.3. If hypothesis $H(\xi)$ holds, then there exists $c_{1}>0$ such that

$$
\vartheta(u)=\|D u\|_{p}^{p}+\int_{\Omega} \xi(z)|u|^{p} d z \geqslant c_{1}\|u\|^{p} \text { for all } u \in W^{1, p}(\Omega) .
$$

Proof. Evidently, $\vartheta \geqslant 0$. Suppose that the lemma is not true. Exploiting the $p$-homogeneity of $\vartheta(\cdot)$ we can find $\left\{u_{n}\right\}_{n \geqslant 1} \subseteq W^{1, p}(\Omega)$ such that

$$
\left\|u_{n}\right\|=1 \text { and } \vartheta\left(u_{n}\right) \leqslant \frac{1}{n} \text { for all } n \in \mathbb{N} .
$$

We may assume that

$$
u_{n} \stackrel{w}{\rightarrow} u \text { in } W^{1, p}(\Omega) \text { and } u_{n} \rightarrow u \text { in } L^{p}(\Omega) \text { as } n \rightarrow \infty .
$$


Clearly, $\vartheta(\cdot)$ is sequentially weakly lower semicontinuous. So, it follows from (2) and (3) that

$$
\begin{array}{cc} 
& \vartheta(u)=0, \\
\Rightarrow \quad & u \equiv \eta \in \mathbb{R} .
\end{array}
$$

If $\eta=0$, then $u_{n} \rightarrow 0$ in $W^{1, p}(\Omega)$, which contradicts (2). So $\eta \neq 0$. Then

$$
\left.0=|\eta|^{p} \int_{\Omega} \xi(z) d z>0 \text { (see [3] and hypothesis } H(\xi)\right),
$$

which is a contradiction. The proof of Lemma 2.3 is now complete.

Let $x \in \mathbb{R}$ and $x^{ \pm}=\max \{ \pm x, 0\}$. Then for all $u \in W^{1, p}(\Omega)$, we set $u^{ \pm}(\cdot)=u(\cdot)^{ \pm}$. We have

$$
u^{ \pm} \in W^{1, p}(\Omega), u=u^{+}-u^{-},|u|=u^{+}+u^{-} .
$$

We denote by $|\cdot|_{N}$ the Lebesgue measure on $\mathbb{R}^{N}$. Given $u, v \in W^{1, p}(\Omega)$ with $u \leqslant v$, define

$$
[u, v]=\left\{y \in W^{1, p}(\Omega): u(z) \leqslant y(z) \leqslant v(z) \text { for almost all } z \in \Omega\right\} .
$$

Also, we denote by $\operatorname{int}_{C^{1}(\bar{\Omega})}[u, v]$ the interior of $[u, v] \cap C^{1}(\bar{\Omega})$ in the $C^{1}(\bar{\Omega})$-norm topology. Finally, if $1<p<\infty$, we denote by $p^{\prime}>1$ the conjugate exponent of $p>1$, that is, $\frac{1}{p}+\frac{1}{p^{\prime}}=1$.

Now we can introduce our hypotheses on $f(z, x, y)$ :

$H(f): f: \Omega \times \mathbb{R} \times \mathbb{R}^{N} \rightarrow \mathbb{R}$ is a Carathéodory function such that $f(z, 0, y)=0$ for almost all $z \in \Omega$ and all $y \in \mathbb{R}^{N}$, and the following properties hold:

(i) there exists a function $w \in W^{1, p}(\Omega) \cap C(\bar{\Omega})$ such that $\Delta_{p} w \in L^{p^{\prime}}(\Omega)$ and

$$
\begin{aligned}
& 0<\hat{c} \leqslant w(z) \text { for all } z \in \bar{\Omega},-\Delta_{p} w(z)+\xi(z) w(z)^{p-1} \geqslant 0 \text { for almost all } z \in \Omega, \\
& w(z)^{-\gamma}+f(z, w(z), y) \leqslant-c^{*}<0 \text { for almost all } z \in \Omega \text { and all } y \in \mathbb{R}^{N},
\end{aligned}
$$

and if $\rho=\|w\|_{\infty}$, there exists $\hat{a}_{\rho} \in L^{\infty}(\Omega)$ such that

$$
|f(z, x, y)| \leqslant \hat{a}_{\rho}(z)\left[1+|y|^{p-1}\right]
$$

for almost all $z \in \Omega$, all $0 \leqslant x \leqslant \rho$, and all $y \in \mathbb{R}^{N}$;

(ii) there exists $\delta_{0}>0$ such that $f(z, x, y) \geqslant \tilde{c}_{\delta}>0$ for almost all $z \in \Omega$ and all $0<\delta \leqslant x \leqslant \delta_{0}$, $y \in \mathbb{R}^{N}$

(iii) there exists $\hat{\xi}_{\rho}>0$ such that for almost all $z \in \Omega$ and all $y \in \mathbb{R}^{N}$ the mapping

$$
x \mapsto f(z, x, y)+\hat{\xi}_{\rho} x^{p-1}
$$

is nondecreasing on $[0, \rho]$, and for almost all $z \in \Omega$, all $0 \leqslant x \leqslant \rho, y \in \mathbb{R}^{N}$, and $t \in(0,1)$, we have

$$
f\left(z, \frac{1}{t} x, y\right) \leqslant \frac{1}{t^{p-1}} f(z, x, y)
$$

Remark 2.1. Our aim is to produce positive solutions and all the above hypotheses concern the positive semi-axis $\mathbb{R}_{+}=[0,+\infty)$. So, for simplicity, we may assume that

$$
f(z, x, y)=0 \text { for almost all } z \in \Omega \text { and all } x \leqslant 0, y \in \mathbb{R}^{N} .
$$

Hypothesis $H(f)(i)$ is satisfied if, for example, there exists $\eta \in(0,+\infty)$ such that $\eta^{-\gamma}+f(z, \eta, y) \leqslant$ $-c^{*}<0$ for almost all $z \in \Omega$ and all $y \in \mathbb{R}^{N}$. Hypotheses $H(f)(i),($ ii $)$ together determine the oscillatory behavior of $f(z, \cdot, y)$ near $0^{+}$. Hypothesis $H(f)($ iii $)$ is satisfied if we set $f(z, x, y)=0$ for almost all $z \in \Omega$ and all $x \geqslant w(z), y \in \mathbb{R}^{N}$ and require that the function $x \mapsto \frac{f(z, x, y)}{x^{p-1}}$ is nonincreasing on $(0, w(z)]$ for almost all $z \in \Omega$ and all $y \in \mathbb{R}^{N}$. 
Example 2.1. The following function satisfies hypotheses $H(f)$. For the sake of simplicity we drop the $z$-dependence and require $\xi(z) \geqslant c_{0}^{*}>0$ for almost all $z \in \Omega$ :

$$
f(z, y)=\left(z^{p-1}-c z^{\tau-1}\right)\left(1+|y|^{p-1}\right)
$$

for all $0 \leqslant x \leqslant 1, y \in \mathbb{R}^{N}$, with $1<p<\tau<\infty$, and $c<2^{\frac{1}{\tau-1}}$.

Finally, we mention that $0<\gamma<1$. When the differential operator is singular (that is, $1<p<2$ ), we require that $\gamma \leqslant(p-1)^{2}$, which is equivalent to saying that $1+\frac{\gamma}{p-1} \leqslant p$.

\section{A Singular PROBLEM}

In this section we deal with the following purely singular Neumann problem:

$$
\left\{\begin{array}{l}
-\Delta_{p} u(z)+\xi(z) u(z)^{p-1}=u(z)^{-\gamma} \text { in } \Omega, \\
\frac{\partial u}{\partial n}=0 \text { on } \partial \Omega, u>0 .
\end{array}\right\}
$$

Recall that $\vartheta: W^{1, p}(\Omega) \rightarrow \mathbb{R}$ is the $C^{1}$-functional defined by

$$
\vartheta(u)=\|D u\|_{p}^{p}+\int_{\Omega} \xi(z)|u|^{p} d z \text { for all } u \in W^{1, p}(\Omega) .
$$

Proposition 3.1. If hypotheses $H(\xi)$ hold, then problem (7) has a unique positive solution $\bar{u} \in D_{+}$.

Proof. Let $\epsilon>0$ and consider the $C^{1}$-functional $\psi_{\epsilon}: W^{1, p}(\Omega) \rightarrow \mathbb{R}$ defined by

$$
\psi_{\epsilon}(u)=\frac{1}{p} \vartheta(u)-\frac{1}{1-\gamma} \int_{\Omega}\left[\left(u^{+}\right)^{p}+\epsilon\right]^{\frac{1-\gamma}{p}} d z \text { for all } u \in W^{1, p}(\Omega) .
$$

Using Lemma 2.3, we obtain

$$
\begin{aligned}
& \psi_{\epsilon}(u) \geqslant \frac{c_{1}}{p}\|u\|^{p}-\frac{1}{1-\gamma} \int_{\Omega}\left(u^{+}\right)^{1-\gamma} d z-c_{2} \text { for some } c_{2}>0 \\
\Rightarrow & \psi_{\epsilon}(\cdot) \text { is coercive. }
\end{aligned}
$$

Using the Sobolev embedding theorem, we can easily see that the functional $\psi_{\epsilon}(\cdot)$ is sequentially weakly lower semicontinuous. So, by the Weierstrass-Tonelli theorem, we can find $u_{\epsilon} \in W^{1, p}(\Omega)$ such that

$$
\psi_{\epsilon}\left(u_{\epsilon}\right)=\inf \left\{\psi_{\epsilon}(u): u \in W^{1, p}(\Omega)\right\}
$$

Let $s \in(0,1)$. Then

$$
\begin{aligned}
\psi_{\epsilon}(s) & <\left(\frac{s^{p}}{p}\|\xi\|_{\infty}-\frac{s^{1-\gamma}}{1-\gamma}\right)|\Omega|_{N}(\text { see hypothesis } H(\xi)) \\
& <\left(\frac{s^{p}}{p}\|\xi\|_{\infty}+\frac{1}{1-\gamma}\left(\epsilon^{\frac{1-\gamma}{p}}-s^{1-\gamma}\right)\right)|\Omega|_{N} .
\end{aligned}
$$

If $s>2 \epsilon^{1 / p}$, then

$$
\begin{aligned}
& \frac{s^{p}}{p}\|\xi\|_{\infty}+\frac{1}{1-\gamma}\left(\epsilon^{\frac{1-\gamma}{p}}-s^{1-\gamma}\right) \\
< & \frac{s^{p}}{p}\|\xi\|_{p}-\frac{s^{1-\gamma}}{1-\gamma}\left(1-\frac{1}{2^{1-\gamma}}\right)=\tau(s) .
\end{aligned}
$$

Recall that $s \in(0,1)$ and note that $0<1-\gamma<1<p$. So, we can find small enough $\hat{s} \in(0,1)$ such that

$$
\tau(\hat{s})<0
$$


Then (9), (10), (11) imply that for small enough $\epsilon \in\left(0,\left(\frac{\hat{s}}{2}\right)^{p}\right)$, we have

$$
\begin{aligned}
& \psi_{\epsilon}(\hat{s})<\psi_{\epsilon}(0)=-\frac{1}{1-\gamma} \epsilon^{\frac{1-\gamma}{p}}|\Omega|_{N}, \\
\Rightarrow & \psi_{\epsilon}\left(u_{\epsilon}\right)<\psi_{\epsilon}(0)(\operatorname{see}(8)) \\
\Rightarrow & u_{\epsilon} \neq 0 .
\end{aligned}
$$

From (8) we have

$$
\begin{aligned}
& \psi_{\epsilon}^{\prime}\left(u_{\epsilon}\right)=0 \\
\Rightarrow & \left\langle A\left(u_{\epsilon}\right), h\right\rangle+\int_{\Omega} \xi(z)\left|u_{\epsilon}\right|^{p-2} u_{\epsilon} h d z=\int_{\Omega}\left(u^{+}\right)^{p-1}\left[\left(u^{+}\right)^{p}+\epsilon\right]^{\frac{1-(\gamma+p)}{p}} h d z
\end{aligned}
$$

for all $h \in W^{1, p}(\Omega)$.

In $(12)$ we choose $h=-u_{\epsilon}^{-} \in W^{1, p}(\Omega)$. We obtain

$$
\begin{aligned}
& \vartheta\left(u_{\epsilon}^{-}\right)=0, \\
\Rightarrow & \left.c_{1}\left\|u_{\epsilon}^{-}\right\|^{p} \leqslant 0 \text { (see Lemma } 2.3\right), \\
\Rightarrow & u_{\epsilon} \geqslant 0, u_{\epsilon} \neq 0 .
\end{aligned}
$$

From (12), we have

$$
\left\{\begin{array}{l}
-\Delta_{p} u_{\epsilon}(z)+\xi(z) u_{\epsilon}(z)^{p-1}=u_{\epsilon}(z)^{p-1}\left[u_{\epsilon}(z)^{p}+\epsilon\right]^{\frac{1-(\gamma+p)}{p}} \text { for almost all } z \in \Omega, \\
\frac{\partial u_{\epsilon}}{\partial n}=0 \text { on } \partial \Omega
\end{array}\right\}
$$

(see Papageorgiou \& Rădulescu [17]).

By (13) and Proposition 7 of Papageorgiou \& Rădulescu [18], we have

$$
u_{\epsilon} \in L^{\infty}(\Omega) \text {. }
$$

Then, invoking Theorem 2 of Lieberman [14], we obtain

$$
u_{\epsilon} \in C_{+} \backslash\{0\} \text {. }
$$

From (13) and hypothesis $H(\xi)$, we have

$$
\begin{aligned}
& \Delta_{p} u_{\epsilon}(z) \leqslant\|\xi\|_{\infty} u_{\epsilon}(z)^{p-1} \text { for almost all } z \in \Omega, \\
\Rightarrow & u_{\epsilon} \in D_{+} \text {by the nonlinear maximum principle }
\end{aligned}
$$

(see Gasinski \& Papageorgiou [2, p. 738] and Pucci \& Serrin [25, p. 120]).

So, for small enough $\epsilon>0$, say $\epsilon \in\left(0, \epsilon_{0}\right)$, we obtain a solution $u_{\epsilon} \in D_{+}$for problem (13).

Claim 3.1. $\left\{u_{\epsilon}\right\}_{\epsilon \in\left(0, \epsilon_{0}\right)} \subseteq W^{1, p}(\Omega)$ is bounded.

We argue by contradiction. So, suppose that the claim is not true. Then we can find $\left\{\epsilon_{n}\right\}_{n \geqslant 1} \subseteq\left(0, \epsilon_{0}\right)$ and corresponding solutions $\left\{u_{n}=u_{\epsilon_{n}}\right\}_{n \geqslant 1} \subseteq D_{+}$of (13) such that

$$
\left\|u_{n}\right\| \rightarrow \infty \text { as } n \rightarrow \infty \text {. }
$$

Let $y_{n}=\frac{u_{n}}{\left\|u_{n}\right\|}, n \in \mathbb{N}$. Then

$$
\left\|y_{n}\right\|=1 \text { and } y_{n} \geqslant 0 \text { for all } n \in \mathbb{N} \text {. }
$$

From (12), we obtain

$$
\left\langle A\left(y_{n}\right), h\right\rangle+\int_{\Omega} \xi(z) y_{n}^{p-1} h d z=\int_{\Omega} y_{n}^{p-1}\left[u_{n}^{p}+\epsilon_{n}\right]^{\frac{1-(\gamma+p)}{p}} h d z
$$

for all $h \in W^{1, p}(\Omega), n \in \mathbb{N}$. 
In (16) we choose $h=y_{n} \in W^{1, p}(\Omega)$. Then

$$
\vartheta\left(y_{n}\right)=\int_{\Omega} \frac{y_{n}^{p}}{\left[u_{n}^{p}+\epsilon_{n}\right]^{\frac{p+\gamma-1}{p}}} d z \text { for all } n \in \mathbb{N} .
$$

From the first part of the proof, we know that these solutions $u_{n}$ can be generated by applying the direct method of the calculus of variations to the functionals $\psi_{\epsilon_{n}}(\cdot)$ and we get

$$
\begin{aligned}
& \psi_{\epsilon_{n}}\left(u_{n}\right)<0 \text { for all } n \in \mathbb{N}, \\
\Rightarrow & \vartheta\left(u_{n}\right)-\frac{p}{1-\gamma} \int_{\Omega}\left[u_{n}^{p}+\epsilon_{n}\right]^{\frac{1-\gamma}{p}} d z<0 \text { for all } n \in \mathbb{N} .
\end{aligned}
$$

It follows from (17) and (18) that

$$
\begin{aligned}
\int_{\Omega} \frac{y_{n}^{p}}{\left[u_{n}^{p}+\epsilon_{n}\right]^{\frac{p+\gamma-1}{p}}} d z & <\frac{p}{1-\gamma} \int_{\Omega} \frac{\left[u_{n}^{p}+\epsilon_{n}\right]^{\frac{1-\gamma}{p}}}{\left\|u_{n}\right\|^{p}} d z \\
& \left.\leqslant \frac{p}{1-\gamma} \int_{\Omega} \frac{u_{n}^{1-\gamma}+\epsilon_{n}^{\frac{1-\gamma}{p}}}{\left\|u_{n}\right\|^{p}} d z \rightarrow 0 \text { as } n \rightarrow \infty \text { (see }(14)\right) .
\end{aligned}
$$

Then by (17) and Lemma 2.3, we have

$$
\begin{aligned}
& c_{1}\left\|y_{n}\right\|^{p} \leqslant \int_{\Omega} \frac{y_{n}^{p}}{\left[u_{n}^{p}+\epsilon_{n}\right]^{\frac{p+\gamma-1}{p}}} d z, \\
\Rightarrow & y_{n} \rightarrow 0 \text { in } W^{1, p}(\Omega) \text { as } n \rightarrow \infty(\sec (19)),
\end{aligned}
$$

which contradicts (15). This proves the claim.

Consider a sequence $\left\{\epsilon_{n}\right\}_{n \geqslant 1} \subseteq\left(0, \epsilon_{0}\right)$ such that $\epsilon_{n} \rightarrow 0^{+}$. As before, let $\left\{u_{n}=u_{\epsilon_{n}}\right\}_{n} \geqslant 1 \subseteq D_{+}$ be the corresponding solutions. On account of the claim, we may assume that

$$
u_{n} \stackrel{w}{\rightarrow} \bar{u} \text { in } W^{1, p}(\Omega) \text { and } u_{n} \rightarrow \bar{u} \text { in } L^{p}(\Omega) \text { as } n \rightarrow \infty, \bar{u} \geqslant 0 .
$$

We know that

$$
\begin{aligned}
& \left\langle A\left(u_{n}\right), h\right\rangle+\int_{\Omega} \xi(z) u_{n}^{p-1} h d z=\int_{\Omega} \frac{u_{n}^{p-1}}{\left[u_{n}^{p}+\epsilon_{n}\right]^{\frac{p+\gamma-1}{p}}} h d z \\
& \text { for all } h \in W^{1, p}(\Omega), n \in \mathbb{N} \text {. }
\end{aligned}
$$

Choosing $h=u_{n} \in W^{1, p}(\Omega)$ in (18), we obtain

$$
-\vartheta\left(u_{n}\right)+\int_{\Omega} \frac{u_{n}^{p}}{\left[u_{n}^{p}+\epsilon_{n}\right]^{\frac{p+\gamma-1}{p}}} d z=0 \text { for all } n \in \mathbb{N} .
$$

Moreover, from the first part of the proof (see (11)), we have

$$
\vartheta\left(u_{n}\right)-\frac{p}{1-\gamma} \int_{\Omega}\left[u_{n}^{p}+\epsilon_{n}\right]^{\frac{1-\gamma}{p}} d z \leqslant-c_{2}<0 \text { for all } n \in \mathbb{N} .
$$

We add (22) and (23) and obtain

$$
\begin{aligned}
0 \leqslant \int_{\Omega} \frac{u_{n}^{p}}{\left[u_{n}^{p}+\epsilon_{n}\right]^{\frac{p+\gamma-1}{p}}} d z & \leqslant-c_{2}+\frac{p}{1-\gamma} \int_{\Omega}\left[u_{n}^{p}+\epsilon_{n}\right]^{\frac{1-\gamma}{p}} d z \\
& \leqslant-c_{2}+\frac{p}{1-\gamma} \int_{\Omega}\left[u_{n}^{1-\gamma}+\epsilon_{n}^{\frac{1-\gamma}{n}}\right] d z \text { for all } n \in \mathbb{N} .
\end{aligned}
$$

If $\bar{u}=0($ see $(20))$, then

$$
\int_{\Omega}\left[u_{n}^{1-\gamma}+\epsilon_{n^{\frac{1-\gamma}{n}}}\right] d z \rightarrow 0 \text { as } n \rightarrow \infty .
$$

This together with (24) leads to a contradiction. Therefore

$$
\bar{u} \neq 0 \text {. }
$$


On account of (20) and by passing to a further subsequence if necessary, we may assume that

$$
\left.\begin{array}{l}
u_{n}(z) \rightarrow \bar{u}(z) \text { for almost all } z \in \Omega \text { as } n \rightarrow \infty, \\
0 \leqslant u_{n}(z) \leqslant k(z) \text { for almost all } z \in \Omega \text { and all } n \in \mathbb{N}, \text { with } k \in L^{p}(\Omega) .
\end{array}\right\}
$$

We can always assume that

$$
\max \left\{1, \epsilon_{0}\right\} \leqslant k(z) \text { for almost all } z \in \Omega .
$$

For every $n \in \mathbb{N}$, we introduce the following measurable subsets of $\Omega$

$$
\Omega_{n}^{1}=\left\{z \in \Omega:\left(u_{n}-\bar{u}\right)(z)>0\right\} \text { and } \Omega_{n}^{2}=\left\{z \in \Omega:\left(u_{n}-\bar{u}\right)(z)<0\right\}, n \in \mathbb{N} .
$$

Then we have

$$
\begin{aligned}
& \int_{\Omega} \frac{u_{n}^{p-1}}{\left[u_{n}^{p}+\epsilon_{n}\right]^{\frac{p+\gamma-1}{p}}}\left(u_{n}-\bar{u}\right) d z \\
= & \int_{\Omega_{n}^{1}} \frac{u_{n}^{p-1}}{\left[u_{n}^{p}+\epsilon_{n}\right]^{\frac{p+\gamma-1}{p}}}\left(u_{n}-\bar{u}\right) d z+\int_{\Omega_{n}^{2}} \frac{u_{n}^{p-1}}{\left[u_{n}^{p}+\epsilon_{n}\right]^{\frac{p+\gamma-1}{p}}}\left(u_{n}-\bar{u}\right) d z \\
\leqslant & \int_{\Omega_{n}^{1}} \frac{u_{n}-\bar{u}}{u_{n}^{\gamma}} d z+\int_{\Omega_{n}^{2}} \frac{1}{2 k^{\gamma}}\left(\frac{u_{n}}{k}\right)^{p-1}\left(u_{n}-\bar{u}\right) d z \text { for all } n \in \mathbb{N}(\text { see }(25),(26)) .
\end{aligned}
$$

From (25) we know that

$$
\begin{aligned}
& 0 \leqslant \bar{u}(z) \leqslant k(z) \text { for almost all } z \in \Omega, \\
& -u_{n}(z)^{-\gamma} \leqslant-k(z)^{-\gamma} \text { for almost all } z \in \Omega \text { and all } n \in \mathbb{N} .
\end{aligned}
$$

It follows from (28), (29) that

$$
-\bar{u}(z) u_{n}(z)^{-\gamma} \leqslant-k(z)^{1-\gamma} \text { for almost all } z \in \Omega \text { and all } n \in \mathbb{N} .
$$

Then for all $n \in \mathbb{N}$ we have

$$
\begin{aligned}
& \int_{\Omega_{n}^{1}} \frac{u_{n}-\bar{u}}{u_{n}^{\gamma}} d z=\int_{\Omega_{n}^{1}}\left[u_{n}^{1-\gamma}-\bar{u} u_{n}^{-\gamma}\right] d z \\
& \text { for all } n \in \mathbb{N}(\operatorname{see}(25),(30)), \\
\Rightarrow & \limsup _{n \rightarrow \infty} \int_{\Omega_{n}^{1}} \frac{u_{n}-\bar{u}}{u_{n}^{\gamma}} d z \leqslant 0 .
\end{aligned}
$$

Also, from (25) and (20), we can see that

$$
\int_{\Omega_{n}^{2}} \frac{1}{2 k^{\gamma}}\left(\frac{u_{n}}{k}\right)^{p-1}\left(u_{n}-\bar{u}\right) d z \rightarrow 0 \text { as } n \rightarrow \infty .
$$

We return to (27), pass to the limit as $n \rightarrow \infty$, and use (31) and (32). We obtain

$$
\limsup _{n \rightarrow \infty} \int_{\Omega} \frac{u_{n}^{p-1}}{\left[u_{n}^{p}+\epsilon_{n}\right]^{\frac{p+\gamma-1}{p}}}\left(u_{n}-\bar{u}\right) d z \leqslant 0 .
$$

In $(21)$ we choose $h=u_{n}-\bar{u} \in W^{1, p}(\Omega)$. Then

$$
\begin{aligned}
& \left\langle A\left(u_{n}\right), u_{n}-\bar{u}\right\rangle+\int_{\Omega} \xi(z) u_{n}^{p-1}\left(u_{n}-\bar{u}\right) d z=\int_{\Omega} \frac{u_{n}^{p-1}}{\left[u_{n}^{p}+\epsilon_{n}\right]^{\frac{p+\gamma-1}{p}}}\left(u_{n}-\bar{u}\right) d z \\
& \text { for all } n \in \mathbb{N}, \\
\Rightarrow \quad & \limsup _{n \rightarrow \infty}\left\langle A\left(u_{n}\right), u_{n}-\bar{u}\right\rangle \leqslant 0(\operatorname{see}(20),(33)), \\
\Rightarrow \quad & \left.u_{n} \rightarrow \bar{u} \text { in } W^{1, p}(\Omega) \text { (see Proposition } 2.2\right), \bar{u} \geqslant 0, \bar{u} \neq 0 .
\end{aligned}
$$

Using in (12) as a test function

$$
h=\frac{u_{n}^{p-1}}{\left(u_{n}^{p}+\epsilon_{n}\right)^{\frac{p+\gamma-1}{p}} \frac{p^{\prime}}{p}} \in W^{1, p}(\Omega)
$$


(recall that $u_{n} \in D_{+}$) and our hypothesis on $\gamma$, we can infer that

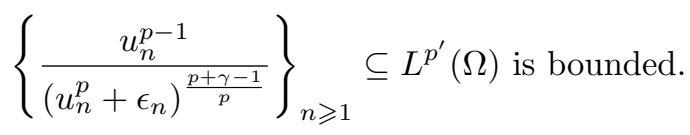

Also, we have

$$
\frac{u_{n}^{p}}{\left(u_{n}^{p-1}+\epsilon_{n}\right)^{\frac{p+\gamma-1}{p}}} \rightarrow \bar{u}^{-\gamma} \text { for almost all } z \in \Omega(\text { see }(25)) .
$$

Then Problem 1.19 in Gasinski \& Papageorgiou [4, p. 46] implies that

$$
\begin{aligned}
& \frac{u_{n}^{p-1}}{\left(u_{n}^{p}+\epsilon_{n}\right)^{\frac{p+\gamma-1}{p}}} \stackrel{w}{\rightarrow} \bar{u}^{-\gamma} \text { in } L^{p^{\prime}}(\Omega), \\
\Rightarrow & \int_{\Omega} \frac{u_{n}^{p-1}}{\left[u_{n}^{p}+\epsilon_{n}\right]^{\frac{p+\gamma-1}{p}}} h d z \rightarrow \int_{\Omega} \bar{u}^{-\gamma} h d z \text { for all } h \in W^{1, p}(\Omega) .
\end{aligned}
$$

Passing to the limit as $n \rightarrow \infty$ in (21) and using (34) and (35), we obtain

$$
\langle A(\bar{u}), h\rangle+\int_{\Omega} \xi(z) \bar{u}^{p-1} h d z=\int_{\Omega} \bar{u}^{-\gamma} h d z \text { for all } h \in W^{1, p}(\Omega)
$$

In (36) we first choose $h=\frac{1}{\left[\bar{u}^{p}+\delta\right]^{\frac{p-1}{p}}} \in W^{1, p}(\Omega), \delta>0$. Then

$$
\begin{aligned}
& \int_{\Omega} \xi(z) \frac{\bar{u}^{p-1}}{\left[\bar{u}^{p}+\delta\right]^{\frac{p-1}{p}}} d z \geqslant \int_{\Omega} \frac{\bar{u}^{-\gamma}}{\left[\bar{u}^{p}+\delta\right]^{\frac{p-1}{p}}} d z, \\
& \left.\int_{\Omega} \frac{\bar{u}^{-\gamma}}{\left[\bar{u}^{p}+\delta\right]^{\frac{p-1}{p}}} d z \leqslant|| \xi \|_{\infty}|\Omega|_{N} \text { (see hypothesis } H(\xi)\right) .
\end{aligned}
$$

We let $\delta \rightarrow 0^{+}$and use Fatou's lemma. Then

$$
\int_{\Omega} \frac{1}{\bar{u}^{p+\gamma-1}} d z \leqslant\|\xi\|_{\infty}|\Omega|_{N}
$$

Next, we choose in $(36) h=\frac{1}{\left[\bar{u}^{p}+\gamma\right]^{\frac{2(p-1)+\gamma}{p}}} \in W^{1, p}(\Omega)$. Reasoning as above, we obtain via Fatou's lemma as $\delta \rightarrow 0^{+}$

$$
\begin{aligned}
\int_{\Omega} \frac{\bar{u}^{-\gamma}}{\bar{u}^{2(p-1)+\gamma}} d z=\int_{\Omega} \frac{1}{\bar{u}^{2(p+\gamma-1)}} d z & \leqslant \int_{\Omega} \xi(z) \frac{\bar{u}^{p-1}}{\bar{u}^{2(p-1)+\gamma}} d z \\
& =\int_{\Omega} \xi(z) \frac{1}{\bar{u}^{p+\gamma-1}} d z \\
& \leqslant\|\xi\|_{\infty}^{2}|\Omega|_{N}(\text { see }(37)) .
\end{aligned}
$$

Continuing in this way, we obtain

$$
\int_{\Omega} \frac{1}{\bar{u}^{k(p+\gamma-1)}} d z \leqslant\|\xi\|_{\infty}^{k}|\Omega|_{N} \text { for all } k \in \mathbb{N} .
$$

Therefore we can infer that

$$
\begin{aligned}
& \bar{u}^{-(p+\gamma-1)} \in L^{\tau}(\Omega) \text { for all } \tau \geqslant 1, \\
& \limsup _{\tau \rightarrow+\infty}\left\|\bar{u}^{-(p+\gamma-1)}\right\|_{\tau}<+\infty .
\end{aligned}
$$

Then Problem 3.104 in Gasinski \& Papageorgiou [3, p. 477] implies that

$$
\bar{u}^{-(p+\gamma-1)} \in L^{\infty}(\Omega) .
$$

Note that

$$
\bar{u}^{-\gamma}=\bar{u}^{-(p+\gamma-1)} \bar{u}^{p-1} .
$$


Therefore from (36) and Proposition 7 of Papageorgiou \& Rădulescu [18], we have

$$
\bar{u} \in L^{\infty}(\Omega) .
$$

Invoking Theorem 2 of Lieberman [14], we have

$$
\bar{u} \in C_{+} \backslash\{0\} .
$$

It follows by (36) that

$$
-\Delta_{p} \bar{u}(z)+\xi(z) \bar{u}(z)^{p-1}=\bar{u}(z)^{-\gamma} \text { for almost all } z \in \Omega, \frac{\partial \bar{u}}{\partial n}=0 \text { on } \partial \Omega
$$

(see Papageorgiou \& Rădulescu [17]),

$\Rightarrow \Delta_{p} \bar{u}(z) \leqslant\|\xi\|_{\infty} \bar{u}(z)^{p-1}$ for almost all $z \in \Omega$,

$\Rightarrow \bar{u} \in D_{+}$(by the nonlinear maximum principle (see $([2$, p. 738] and [25, p. 120]))).

Finally, we can show that the positive solution is unique. Suppose that $\bar{u}_{0} \in W^{1, p}(\Omega)$ is another positive solution of (7). Again we have $\bar{u}_{0} \in D_{+}$. Also

$$
\begin{aligned}
0 & \leqslant\left\langle A(\bar{u})-A\left(\bar{u}_{0}\right), \bar{u}-u_{0}\right\rangle+\int_{\Omega} \xi(z)\left(\bar{u}^{p-1}-\bar{u}_{0}^{p-1}\right)\left(\bar{u}-\bar{u}_{0}\right) d z \\
& =\int_{\Omega}\left(\bar{u}^{-\gamma}-\bar{u}_{0}^{-\gamma}\right)\left(\bar{u}-\bar{u}_{0}\right) d z \leqslant 0, \\
\Rightarrow \quad \bar{u} & \left.=\bar{u}_{0} \text { (the function } x \mapsto \frac{1}{x^{\gamma}} \text { is strictly decreasing on }(0,+\infty)\right) .
\end{aligned}
$$

This proves the uniqueness of the positive solution $\bar{u} \in D_{+}$of (7) and thus completes the proof of Proposition 3.1.

\section{Existence of Positive solutions}

Let $\bar{u} \in D_{+}$be the unique positive solution of (7) produced by Proposition 3.1. We choose $t \in(0,1)$ small enough such that

$$
\left.\tilde{u}=t \bar{u} \leqslant \min \left\{\hat{c}, \delta_{0}\right\} \text { on } \bar{\Omega} \text { (see hypotheses } H(f)(i),(i i)\right) .
$$

Then given $\tilde{v} \in W^{1, p}(\Omega)$, we have

$$
\begin{aligned}
-\Delta_{p} \tilde{u}(z)+\xi(z) \tilde{u}(z)^{p-1} & =t^{p-1}\left[-\Delta_{p} \bar{u}(z)+\xi(z) \bar{u}(z)^{p-1}\right] \\
& =t^{p-1} \bar{u}(z)^{-\gamma}(\text { see }(39)) \\
& \leqslant \tilde{u}(z)^{-\gamma}+f(z, \tilde{u}(z), D v(z)) \text { for almost all } z \in \Omega
\end{aligned}
$$

(see $(40)$ and hypothesis $H(f)(i i))$.

Given $v \in C^{1}(\bar{\Omega})$, we consider the following nonlinear auxiliary Neumann problem:

$$
\left\{\begin{array}{l}
-\Delta_{p} u(z)+\xi(z) u(z)^{p-1}=u(z)^{-\gamma}+f(z, u(z), D v(z)) \text { in } \Omega, \\
\frac{\partial u}{\partial n}=0 \text { on } \partial \Omega, u>0 .
\end{array}\right\}
$$

Proposition 4.1. If hypotheses $H(\xi), H(f)$ hold, then for every $v \in C^{1}(\bar{\Omega})$ problem (42) has a solution $u_{v} \in[\tilde{u}, w] \cap C^{1}(\bar{\Omega})$, with $w(\cdot)$ being the function from hypothesis $H(f)(i)$.

Proof. We introduce the following truncation of the reaction term in problem (1):

$$
\hat{f}_{v}(z, x)=\left\{\begin{array}{l}
\tilde{u}(z)^{-\gamma}+f(z, \tilde{u}(z), D v(z)) \text { if } x<\tilde{u}(z) \\
x^{-\gamma}+f(z, x, D v(z)) \text { if } \tilde{u}(z) \leqslant x \leqslant w(z) \\
w(z)^{-\gamma}+f(z, w(z), D v(z)) \text { if } w(z)<x
\end{array}\right.
$$


Evidently, $\hat{f}_{v}(\cdot, \cdot)$ is a Carathéodory function. We set $\hat{F}_{v}(z, x)=\int_{0}^{x} \hat{f}_{v}(z, s) d s$ and consider the $C^{1}$-functional $\hat{\varphi}_{v}: W^{1, p}(\Omega) \rightarrow \mathbb{R}$ defined by

$$
\hat{\varphi}_{v}(u)=\frac{1}{p} \vartheta(u)-\int_{\Omega} \hat{F}_{v}(z, u(z)) d z \text { for all } u \in W^{1, p}(\Omega) .
$$

It is clear from (43) that $\hat{\varphi}_{v}(\cdot)$ is coercive. Also, it is sequentially weakly lower semicontinuous. So, by the Weierstrass-Tonelli theorem, we can find $u_{v} \in W^{1, p}(\Omega)$ such that

$$
\begin{aligned}
& \hat{\varphi}_{v}\left(u_{v}\right)=\inf \left\{\hat{\varphi}_{v}(u): u \in W^{1, p}(\Omega)\right\}, \\
\Rightarrow & \hat{\varphi}_{v}^{\prime}\left(u_{v}\right)=0 \\
\Rightarrow & \left\langle A\left(u_{v}\right), h\right\rangle+\int_{\Omega} \xi(z)\left|u_{v}\right|^{p-2} u_{v} h d z=\int_{\Omega} \hat{f}_{v}\left(z, u_{v}\right) h d z \text { for all } h \in W^{1, p}(\Omega) .
\end{aligned}
$$

In (44) we first choose $h=\left(\tilde{u}-u_{v}\right)^{+} \in W^{1, p}(\Omega)$. We have

$$
\begin{aligned}
& \left\langle A\left(u_{v}\right),\left(\tilde{u}-u_{v}\right)^{+}\right\rangle+\int_{\Omega} \xi(z)\left|u_{v}\right|^{p-2} u_{v}\left(\tilde{u}-u_{v}\right)^{+} d z \\
& =\int_{\Omega}\left[\tilde{u}^{-\gamma}+f(z, \tilde{u}, D v)\right]\left(\tilde{u}-u_{v}\right)^{+} d z(\text { see }(43)) \\
& \geqslant\left\langle A(\tilde{u}),\left(\tilde{u}-u_{v}\right)^{+}\right\rangle+\int_{\Omega} \xi(z) \tilde{u}^{p-1}\left(\tilde{u}-u_{v}\right)^{+} d z(\text { see }(41)), \\
\Rightarrow & 0 \geqslant\left\langle A(\tilde{u})-A\left(u_{v}\right),\left(\tilde{u}-u_{v}\right)^{+}\right\rangle+\int_{\Omega} \xi(z)\left(\tilde{u}^{p-1}-\left|u_{v}\right|^{p-2} u_{v}\right)\left(\tilde{u}-u_{v}\right)^{+} d z, \\
\Rightarrow & \tilde{u} \leqslant u_{v} .
\end{aligned}
$$

Next, we choose in (44) $h=\left(u_{v}-w\right)^{+} \in W^{1, p}(\Omega)$. Then

$$
\begin{aligned}
& \left\langle A\left(u_{v}\right),\left(u_{v}-w\right)^{+}\right\rangle+\int_{\Omega} \xi(z) u_{v}^{p-1}\left(u_{v}-w\right)^{+} d z(\text { see }(45)) \\
& =\int_{\Omega}\left[w^{-\gamma}+f(z, w, D v)\right]\left(u_{v}-w\right)^{+} d z(\text { see }(43)) \\
& \left.\leqslant\left\langle A(w),\left(u_{v}-w\right)^{+}\right\rangle+\int_{\Omega} \xi(z) w^{p-1}\left(u_{v}-w\right)^{+} d z \text { (see hypothesis } H(f)(i)\right), \\
\Rightarrow & \left\langle A\left(u_{v}\right)-A(w),\left(u_{v}-w\right)^{+}\right\rangle+\int_{\Omega} \xi(z)\left(u_{v}^{p-1}-w^{p-1}\right)\left(u_{v}-w\right)^{+} d z \leqslant 0, \\
\Rightarrow & u_{v} \leqslant w .
\end{aligned}
$$

It follows from (45) and (46) that

$$
u_{v} \in[\tilde{u}, w] .
$$

On account of (47), (43) and (44), we have

$$
\begin{aligned}
& -\Delta_{p} u_{v}(z)+\xi(z) u_{v}(z)^{p-1}=u_{v}(z)^{-\gamma}+f\left(z, u_{v}(z), D v(z)\right) \text { for almost all } z \in \Omega, \\
& \frac{\partial u_{v}}{\partial n}=0 \text { on } \partial \Omega \\
& \text { (see Papageorgiou \& Rădulescu [17]). }
\end{aligned}
$$

From (48) and Papageorgiou \& Rădulescu [18, Proposition 7], we have

$$
u_{v} \in L^{\infty}(\Omega) \text {. }
$$

Then Theorem 2 of Lieberman [14] implies that $u_{v} \in D_{+}$. Therefore

$$
u_{v} \in[\tilde{u}, w] \cap C^{1}(\bar{\Omega}) .
$$

The proof of Proposition 4.1 is now complete. 
We introduce the solution set

$$
S_{v}=\left\{u \in W^{1, p}(\Omega): u \text { is a solution of }(42), u \in[\tilde{u}, w]\right\} .
$$

By Proposition 4.1, we have

$$
\emptyset \neq S_{v} \subseteq[\tilde{u}, v] \cap C^{1}(\bar{\Omega}) .
$$

In fact, we have the following stronger result for the elements of $S_{v}$.

Proposition 4.2. If hypotheses $H(\xi), H(f)$ hold and $u \in S_{v}$, then $u \in \operatorname{int}_{C^{1}(\bar{\Omega})}[\tilde{u}, w]$.

Proof. Let $\tilde{\rho}=\min _{\bar{\Omega}} \tilde{u}>0$ (recall that $\tilde{u} \in D_{+}$). So, we can increase $\hat{\xi}_{\rho}>0$ postulated by hypothesis $H(f)($ iii $)$ in order to guarantee that for almost all $z \in \Omega$, the function

$$
x \mapsto x^{-\gamma}+f(z, x, D v(z))+\hat{\xi}_{p} x^{p-1}
$$

is nondecreasing on $[\tilde{\rho}, \rho] \subseteq \mathbb{R}_{+}$.

Let $\delta>0$ and set $\tilde{u}^{\delta}=\tilde{u}+\delta \in D_{+}$. Then

$$
\begin{aligned}
& -\Delta_{p} \tilde{u}^{\delta}+\left(\xi(z)+\hat{\xi}_{\rho}\right)\left(\tilde{u}^{\delta}\right)^{p-1} \\
& \leqslant-\Delta_{p} \tilde{u}+\left(\xi(z)+\hat{\xi}_{\rho}\right) \tilde{u}^{p-1}+\lambda(\delta) \text { with } \lambda(\delta) \rightarrow 0^{+} \text {as } \delta \rightarrow 0^{+} \\
& \leqslant \tilde{u}^{-\gamma}+f(z, \tilde{u}, D v)+\hat{\xi}_{\rho} \tilde{u}^{p-1} \text { for } \delta>0 \text { small enough } \\
& \left(\text { since } f(z, \tilde{u}, D v) \geqslant \tilde{c}_{\tilde{\rho}}>0 \text { for almost all } z \in \Omega, \text { see } H(f)(i)\right) \\
& \left.\leqslant u^{-\gamma}+f(z, u, D v)+\hat{\xi}_{\rho} u^{p-1} \text { (since } \tilde{u} \leqslant u\right) \\
& =-\Delta_{p} u+\left(\xi(z)+\hat{\xi}_{\rho}\right) u^{p-1} \text { for almost all } z \in \Omega\left(\text { since } u \in S_{v}\right), \\
\Rightarrow & \tilde{u}^{\delta} \leqslant u \text { for small enough } \delta>0, \\
\Rightarrow & u-\tilde{u} \in D_{+} .
\end{aligned}
$$

Similarly, for $\delta>0$ let $u^{\delta}=u+\delta \in D_{+}$. Then

$$
\begin{aligned}
& -\Delta_{p} u^{\delta}+\left(\xi(z)+\hat{\xi}_{\rho}\right)\left(u^{\delta}\right)^{p-1} \\
& \leqslant-\Delta_{p} u+\left(\xi(z)+\hat{\xi}_{\rho}\right) u^{p-1}+\tilde{\lambda}(\lambda) \text { with } \tilde{\lambda}(\delta) \rightarrow 0^{+} \text {as } \delta \rightarrow 0^{+} \\
& =u^{-\gamma}+f(z, u, D v)+\hat{\xi}_{\rho} u^{p-1}+\tilde{\lambda}(\delta)\left(\text { since } u \in S_{v}\right) \\
& \leqslant w^{-\gamma}+f(z, w, D v)+\hat{\xi}_{\rho} u^{p-1}+\tilde{\lambda}(\delta)(\text { since } u \leqslant w) \\
& \leqslant-c^{*}+\tilde{\lambda}(\delta)+\hat{\xi}_{\rho} u^{p-1}(\text { see hypothesis } H(f)(i)) \\
& \leqslant-\Delta_{p} w+\left(\xi(z)+\hat{\xi}_{p}\right) w^{p-1} \text { for almost all } z \in \Omega \text { and for small enough } \delta>0 \\
& \left(\text { since } \tilde{\lambda}(\delta) \rightarrow 0^{+} \text {as } \delta \rightarrow 0^{+} \text {and due to hypothesis } H(f)(i)\right), \\
\Rightarrow \quad & u^{\delta} \leqslant w \text { for small enough } \delta>0, \\
\Rightarrow \quad & (w-u)(z)>0 \text { for all } z \in \bar{\Omega} .
\end{aligned}
$$

Therefore we conclude that

$$
u \in \operatorname{int}_{C^{1}(\bar{\Omega})}[\tilde{u}, w] .
$$

The proof of Proposition 4.2 is now complete.

We can show that $S_{v}$ admits a smallest element, that is, there exists $\hat{u}_{v} \in S_{v}$ such that $\hat{u}_{v} \leqslant u$ for all $u \in S_{v}$.

Proposition 4.3. If hypotheses $H(\xi), H(f)$ hold, then for every $v \in C^{1}(\bar{\Omega})$, the solution set $S_{v}$ admits a smallest element

$$
\hat{u}_{v} \in S_{v}
$$


Proof. Invoking Lemma 3.10 in Hu \& Papageorgiou [12, p. 178], we can find a sequence $\left\{u_{n}\right\}_{n \geqslant 1} \subseteq S_{v}$ such that

$$
\operatorname{essinf} S_{v}=\inf _{n \geqslant 1} u_{n}
$$

For every $n \in \mathbb{N}$, we have

$$
\left\langle A\left(u_{n}\right), h\right\rangle+\int_{\Omega} \xi(z) u_{n}^{p-1} h d z=\int_{\Omega}\left[u_{n}^{-\gamma}+f\left(z, u_{n}, D v\right)\right] h d z
$$

for all $h \in W^{1, p}(\Omega), n \in \mathbb{N}$,

$\tilde{u} \leqslant u_{n} \leqslant w$ for all $n \in \mathbb{N}$.

It follows from (49) and (50) that

$$
\left\{u_{n}\right\}_{n \geqslant 1} \subseteq W^{1, p}(\Omega) \text { is bounded. }
$$

So, we may assume that

$$
u_{n} \stackrel{w}{\rightarrow} \hat{u}_{v} \text { in } W^{1, p}(\Omega) \text { and } u_{n} \rightarrow \hat{u}_{v} \text { in } L^{p}(\Omega) \text { as } n \rightarrow \infty, \hat{u}_{v} \in[\tilde{u}, w] .
$$

In (49) we choose $h=u_{n}-\hat{u}_{v} \in W^{1, p}(\Omega)$, pass to the limit as $n \rightarrow \infty$, and use (51). Then

$$
\begin{aligned}
& \lim _{n \rightarrow \infty}\left\langle A\left(u_{n}\right), u_{n}-\hat{u}_{v}\right\rangle=0 \text { see }(50), \\
\Rightarrow & u_{n} \rightarrow \hat{u}_{v} \text { in } W^{1, p}(\Omega) \text { (see Proposition 2.2). }
\end{aligned}
$$

Therefore, if in (49) we pass to the limit as $n \rightarrow \infty$ and use (52), then

$$
\begin{aligned}
& \left\langle A\left(\hat{u}_{v}\right), h\right\rangle+\int_{\Omega} \xi(z) \hat{u}_{v}^{p-1} h d z=\int_{\Omega}\left[\hat{u}_{v}^{-\gamma}+f\left(z, \hat{u}_{v}, D v\right)\right] h d z \\
& \text { for all } h \in W^{1, p}(\Omega), \\
\Rightarrow \quad & \hat{u}_{v} \in S_{v} \subseteq D_{+} \text {and } \operatorname{essinf} S_{v}=\hat{u}_{v} .
\end{aligned}
$$

The proof of Proposition 4.3 is now complete.

We can define a map $\sigma: C^{1}(\bar{\Omega}) \rightarrow C^{1}(\bar{\Omega})$ by

$$
\sigma(v)=\hat{u}_{v} .
$$

This map is well-defined by Proposition 4.3 and any fixed point of $\sigma(\cdot)$ is a solution of problem (1). To generate a fixed point for $\sigma(\cdot)$, we will use Theorem 2.1 (the Leray-Schauder alternative principle). For this purpose, the next lemma will be useful.

Lemma 4.4. If hypotheses $H(\xi), H(f)$ hold, $\left\{v_{n}\right\}_{n \geqslant 1} \subseteq C^{1}(\bar{\Omega}), v_{n} \rightarrow v$ in $C^{1}(\bar{\Omega})$, and $u \in S_{v}$, then for every $n \in \mathbb{N}$ there exists $u_{n} \in S_{v_{n}}$ such that $u_{n} \rightarrow u$ in $C^{1}(\bar{\Omega})$.

Proof. We consider the following nonlinear Neumann problem

$$
\left\{\begin{array}{l}
-\Delta_{p} y(z)+\xi(z)|y(z)|^{p-2} y(z)=u(z)^{-\gamma}+f\left(z, u(z), D v_{n}(z)\right) \text { in } \Omega, \\
\frac{\partial y}{\partial n}=0 \text { on } \partial \Omega .
\end{array}\right\}
$$

Since $u \in S_{v} \subseteq D_{+}$, we have

$$
\left\{\begin{array}{l}
k_{n}(z)=u(z)^{-\gamma}+f\left(z, u(z), D v_{n}(z)\right) \geqslant 0 \text { for almost all } z \in \Omega \text { and all } n \in \mathbb{N}, \\
\left\{k_{n}\right\}_{n \geqslant 1} \subseteq L^{\infty}(\Omega) \text { is bounded, } k_{n} \neq 0 \text { for all } n \in \mathbb{N} \\
\text { (see hypotheses } H(f)(i),(i i)) .
\end{array}\right\}
$$

In problem (53), the left-hand side determines a maximal monotone coercive operator (see Lemma 2.3 ), which is strictly monotone. Therefore, on account of (54), problem (53) admits a unique solution $y_{n}^{0} \in W^{1, p}(\Omega), y_{n}^{0} \neq 0$. We have for all $n \in \mathbb{N}$

$$
\left\langle A\left(y_{n}^{0}\right), h\right\rangle+\int_{\Omega} \xi(z)\left|y_{n}^{0}\right|^{p-2} y_{n}^{0} h d z=\int_{\Omega} k_{n}(z) h d z \text { for all } h \in W^{1, p}(\Omega) .
$$


In (55) we choose $h=-\left(y_{n}^{0}\right)^{-} \in W^{1, p}(\Omega)$. Then

$$
\begin{aligned}
& \vartheta\left(\left(y_{n}^{0}\right)^{-}\right) \leqslant 0(\text { see }(54)), \\
\Rightarrow & \left.c_{1}\left\|\left(y_{n}^{0}\right)^{-}\right\|^{p} \leqslant 0 \text { (see Lemma } 2.3\right), \\
\Rightarrow & y_{n}^{0} \geqslant 0, y_{n}^{0} \neq 0 \text { for all } n \in \mathbb{N} .
\end{aligned}
$$

Also, it is clear from (54) and (55) that

$$
\left\{y_{n}^{0}\right\}_{n \geqslant 1} \subseteq W^{1, p}(\Omega) \text { is bounded. }
$$

Invoking Proposition 7 of Papageorgiou \& Rădulescu [18], we have

$$
y_{n}^{0} \in L^{\infty}(\Omega) \text { and }\left\|y_{n}^{0}\right\|_{\infty} \leqslant c_{5} \text { for some } c_{5}>0 \text { and all } n \in \mathbb{N} .
$$

Then (53) and Theorem 2 of Lieberman [14] imply that there exist $\alpha \in(0,1)$ and $c_{6}>0$ such that

$$
y_{n}^{0} \in C^{1, \alpha}(\bar{\Omega}) \text { and }\left\|y_{n}^{0}\right\|_{C^{1, \alpha}(\bar{\Omega})} \leqslant c_{6} \text { for all } n \in \mathbb{N} .
$$

Recall that $C^{1, \alpha}(\bar{\Omega})$ is compactly embedded in $C^{1}(\bar{\Omega})$. So, from (57) we see that we can find a subsequence $\left\{y_{n_{k}}^{0}\right\}_{k \geqslant 1}$ of $\left\{y_{n}^{0}\right\}_{n \geqslant 1}$ such that

$$
y_{n_{k}}^{0} \rightarrow y^{0} \text { in } C^{1}(\bar{\Omega}) \text { as } k \rightarrow \infty, y^{0} \geqslant 0 .
$$

Note that

$$
k_{n} \rightarrow k \text { in } L^{p^{\prime}}(\Omega) \text { with } k(z)=u(z)^{-\gamma}+f(z, u(z), D v(z)) .
$$

Using (55) (for the $y_{n_{k}}^{0}$ 's) and (58), (59), we obtain

$$
\begin{aligned}
& \left\langle A\left(y^{0}\right), h\right\rangle+\int_{\Omega} \xi(z)\left(y^{0}\right)^{p-1} h d z=\int_{\Omega} k(z) h d z \text { for all } h \in W^{1, p}(\Omega), \\
\Rightarrow \quad & -\Delta_{p} y^{0}(z)+\xi(z) y^{0}(z)^{p-1}=u(z)^{-\gamma}+f(z, u(z), D v(z)) \text { for almost all } z \in \Omega, \\
& \frac{\partial y^{0}}{\partial n}=0 \text { on } \partial \Omega .
\end{aligned}
$$

Problem (60) admits a unique solution. Since $u \in S_{v}, u$ solves $(60)$ and so $y^{0}=u$. Therefore for the initial sequence we have

$$
y_{n}^{0} \rightarrow u \text { in } C^{1}(\bar{\Omega}) \text { as } n \rightarrow \infty .
$$

Next, we consider the following nonlinear Neumann problem

$$
\left\{\begin{array}{l}
-\Delta_{p} y(z)+\xi(z)|y(z)|^{p-2} y(z)=y_{n}^{0}(z)^{-\gamma}+f\left(z, y_{n}^{0}(z), D v_{n}(z)\right) \text { in } \Omega, \\
\frac{\partial y}{\partial n}=0 \text { on } \partial \Omega .
\end{array}\right\}
$$

Evidently, this problem has a unique solution $y_{n}^{1} \in D_{+}$and

$$
y_{n}^{1} \rightarrow u \text { in } C^{1}(\bar{\Omega}) \text { as } n \rightarrow \infty(\text { see }(61)) .
$$

Continuing in this way, we produce a sequence $\left\{y_{n}^{k}\right\}_{k, n \in \mathbb{N}}$ such that

$$
\begin{aligned}
& \left\{\begin{array}{l}
-\Delta_{p} y_{n}^{k}(z)+\xi(z) y_{n}^{k}(z)^{p-1}=y_{n}^{k-1}(z)^{-\gamma}+f\left(z, y_{n}^{k-1}(z), D v_{n}(z)\right) \\
\text { for almost all } z \in \Omega, \\
\frac{\partial u_{n}^{k}}{\partial n}=0 \text { on } \partial \Omega, k, n \in \mathbb{N}
\end{array}\right\} \\
& \text { and } y_{n}^{k} \rightarrow u \text { in } C^{1}(\bar{\Omega}) \text { as } n \rightarrow \infty \text { for all } k \in \mathbb{N} \text {. }
\end{aligned}
$$

From (59), (60) and Theorem 2 of Lieberman [14], we can deduce as before that $\left\{y_{n}^{k}\right\}_{k \in \mathbb{N}} \subseteq C^{1}(\bar{\Omega})$ is relatively compact. 
So, we can find a subsequence $\left\{y_{n}^{k_{m}}\right\}_{m \in \mathbb{N}}$ of $\left\{y_{n}^{k}\right\}_{k \in \mathbb{N}}(n \in \mathbb{N}$ is fixed) such that

$$
y_{n}^{k_{m}} \rightarrow \hat{y}_{n} \text { in } C^{1}(\bar{\Omega}), n \in \mathbb{N} .
$$

From (62) in the limit we obtain

$$
\left\{\begin{array}{l}
-\Delta_{p} \hat{y}_{n}(z)+\xi(z) \hat{y}_{n}(z)^{p-1}=\hat{y}_{n}(z)^{-\gamma}+f\left(z, \hat{y}_{n}(z), D v_{n}(z)\right) \text { for almost all } z \in \Omega, \\
\frac{\partial \hat{y}_{n}}{\partial n}=0 \text { on } \partial \Omega .
\end{array}\right\}
$$

Then, using Theorem 2 of Lieberman [14] as before, (63) and the double limit lemma (see Gasinski \& Papageorgiou [3, Problem 1.175, p. 61]) we obtain

$$
\begin{aligned}
& \hat{y}_{n} \rightarrow u \text { in } C^{1}(\bar{\Omega}) \text { as } n \rightarrow \infty, \\
& \text { and } \hat{y}_{n} \in S_{v_{n}} \text { for } n \geqslant n_{0} \text { (see Proposition 4.2). }
\end{aligned}
$$

The proof of Lemma 4.4 is now complete.

Using this lemma we can show that the minimal solution map $\sigma(\cdot)$ is compact.

Proposition 4.5. If hypotheses $H(\xi), H(f)$ hold, then the minimal solution map $\sigma: C^{1}(\bar{\Omega}) \rightarrow$ $C^{1}(\bar{\Omega})$ defined by $\sigma(v)=\hat{u}_{v}$ is compact.

Proof. We first show that $\sigma(\cdot)$ is continuous. To this end, let $v_{n} \rightarrow v$ in $C^{1}(\bar{\Omega})$ and $\hat{u}_{n}=\hat{u}_{v_{n}}=\sigma\left(v_{n}\right)$, $n \in \mathbb{N}$. We have

$$
\left\langle A\left(\hat{u}_{n}\right), h\right\rangle+\int_{\Omega} \xi(z) \hat{u}_{n}^{p-1} h d z=\int_{\Omega}\left[\hat{u}_{n}^{-\gamma}+f\left(z, \hat{u}_{n}, D v_{n}\right)\right] h d z
$$

$$
\text { for all } h \in W^{1, p}(\Omega), n \in \mathbb{N} \text {. }
$$

Choosing $h=\hat{u}_{n} \in W^{1, p}(\Omega)$, we obtain

$$
\begin{aligned}
& \left\|D \hat{u}_{n}\right\|_{p}^{p}+\int_{\Omega} \xi(z) \hat{u}_{p}^{p} d z \leqslant \int_{\Omega} c_{7}\left[\tilde{u}^{-\gamma}+1\right] d z \text { for some } c_{7}>0, \text { and all } n \in \mathbb{N} \\
& \left(\text { since } \tilde{u} \leqslant \hat{u}_{n} \leqslant w \text { for all } n \in \mathbb{N} \text { and due to hypothesis } H(f)(i i)\right), \\
\Rightarrow \quad & \left.c_{1}\left\|\hat{u}_{n}\right\|^{p} \leqslant c_{8} \text { for some } c_{8}>0 \text { and all } n \in \mathbb{N} \text { (see Lemma } 2.3\right), \\
\Rightarrow \quad & \left\{\hat{u}_{n}\right\}_{n \in \mathbb{N}} \subseteq W^{1, p}(\Omega) \text { is bounded. }
\end{aligned}
$$

Invoking Proposition 7 of Papageorgiou \& Rădulescu [18], we have

$$
\left\|\hat{u}_{n}\right\|_{\infty} \leqslant c_{9} \text { for some } c_{9}>0 \text { and all } n \in \mathbb{N} .
$$

Then Theorem 2 of Lieberman [14] implies that we can find $\beta \in(0,1)$ and $c_{10}>0$ such that

$$
\hat{u}_{n} \in C^{1, \beta}(\bar{\Omega}) \text { and }\left\|\hat{u}_{n}\right\|_{C^{1, \beta}(\bar{\Omega})} \leqslant c_{10} \text { for all } n \in \mathbb{N} .
$$

The compact embedding of $C^{1, \beta}(\bar{\Omega})$ into $C^{1}(\bar{\Omega})$ and $(66)$ imply that at least for a subsequence, we have

$$
\hat{u}_{n} \rightarrow \hat{u} \text { in } C^{1}(\bar{\Omega}) \text { as } n \rightarrow \infty .
$$

Passing to the limit as $n \rightarrow \infty$ in (65), we can infer that $\hat{u} \in S_{v}$.

We know that $\sigma(v) \in S_{v}$ and so by Lemma 4.4, we can find $u_{n} \in S_{v_{n}}$ (for all $n \in \mathbb{N}$ ) such that

$$
u_{n} \rightarrow \sigma(v) \text { in } C^{1}(\bar{\Omega}) \text { as } n \rightarrow+\infty .
$$

We have

$$
\begin{aligned}
& \hat{u}_{n} \leqslant u_{n} \text { for all } n \in \mathbb{N}, \\
\Rightarrow \quad & \hat{u} \leqslant \sigma(v), \\
\Rightarrow \quad & \sigma(v)=\hat{u}\left(\text { since } \hat{u} \in S_{v}\right) .
\end{aligned}
$$


So, for the original sequence $\left\{\hat{u}_{n}=\sigma\left(v_{n}\right)\right\}_{n \in \mathbb{N}} \subseteq C^{1}(\bar{\Omega})$, we have

$$
\begin{aligned}
& \sigma\left(v_{n}\right)=\hat{u}_{n} \rightarrow \hat{u}=\sigma(v) \text { in } C^{1}(\bar{\Omega}), \\
\Rightarrow & \sigma(\cdot) \text { is continuous. }
\end{aligned}
$$

Next, let $B \subseteq C^{1}(\bar{\Omega})$ be bounded. As before, we obtain

$$
\begin{aligned}
& \sigma(B) \subseteq W^{1, p}(\Omega) \text { is bounded, } \\
& \Rightarrow \quad \sigma(B) \subseteq L^{\infty}(\Omega) \text { is bounded (see [18]). }
\end{aligned}
$$

Then by Lieberman [14] we conclude that

$$
\overline{\sigma(B)} \subseteq C^{1}(\bar{\Omega}) \text { is compact. }
$$

This proves that the minimal solution map $\sigma(\cdot)$ is compact. The proof of Proposition 4.5 is now complete.

Now using Theorem 2.1 (the Leray-Schauder alternative principle), we will produce a positive smooth solution for problem (1).

Theorem 4.6. If hypotheses $H(\xi), H(f)$ hold, then problem (1) admits a positive solution $u^{*} \in D_{+}$.

Proof. We consider the minimal solution map $\sigma: C^{1}(\bar{\Omega}) \rightarrow C^{1}(\bar{\Omega})$. From Proposition 4.5 we know that $\sigma(\cdot)$ is compact. Let

$$
K=\left\{u \in C^{1}(\bar{\Omega}): u=t \sigma(u), 0<t<1\right\} .
$$

We claim that $K \subseteq C^{1}(\bar{\Omega})$ is bounded. So, let $u \in K$. We have

$$
\frac{1}{t} u=\sigma(u) \text { with } 0<t<1 \text {. }
$$

Then

$$
\begin{aligned}
& \langle A(u), h\rangle+\int_{\Omega} \xi(z) u^{p-1} h d z=t^{p-1} \int_{\Omega}\left[\frac{t^{\gamma}}{u^{\gamma}}+f\left(z, \frac{1}{t} u, D u\right)\right] h d z \\
& \text { for all } h \in W^{1, p}(\Omega) .
\end{aligned}
$$

From (15) (see hypothesis $H(f)(i i i))$, we have

$$
f\left(z, \frac{1}{t} u(z), D u(z)\right) \leqslant \frac{1}{t^{p-1}} f(z, u(z), D u(z)) \text { for almost all } z \in \Omega .
$$

Using (70) in (69) and recalling that $\tilde{u} \leqslant u, 0<t<1$, we obtain

$$
\langle A(u), h\rangle+\int_{\Omega} \xi(z) u^{p-1} h d z \leqslant \int_{\Omega}\left[\frac{1}{\tilde{u}^{\gamma}}+\hat{a}_{0}(z)\right] h d z
$$

for all $h \in W^{1, p}(\Omega)$ and some $\hat{a}_{0} \in L^{\infty}(\Omega)$ (see hypothesis $H(f)(i)$ ).

In (71) we choose $h=u \in W^{1, p}(\Omega)$. Then

$$
\begin{aligned}
& \vartheta(u) \leqslant c_{11} \text { for some } c_{11}>0\left(\text { recall } \tilde{u} \in D_{+}\right), \\
& c_{1}\|u\|^{p} \leqslant c_{11} \text { for all } u \in K(\text { see Lemma } 2.3), \\
\Rightarrow \quad & K \subseteq W^{1, p}(\Omega) \text { is bounded. }
\end{aligned}
$$

Next, as before, the nonlinear regularity theory implies that

$$
K \subseteq C^{1}(\bar{\Omega}) \text { is bounded (in fact, relatively compact). }
$$

So, we can apply Theorem 2.1 (the Leray-Schauder principle) and produce $u^{*} \in C^{1}(\bar{\Omega})$ such that $u^{*}=\sigma\left(u^{*}\right)$. Therefore $u^{*} \in D_{+}$is a positive smooth solution of problem (1). The proof of Theorem 4.6 is now complete.

Acknowledgements. This research was supported by the Slovenian Research Agency grants P1-0292, J1-8131, N1-0064, N1-0083, and N1-0114. 


\section{REFERENCES}

[1] D. de Figueiredo, M. Girardi, M. Matzeu, Semilinear elliptic equations with dependence on the gradient via mountain-pass techniques, Differential Integral Equations 17 (2004), 119-126.

[2] L. Gasinski, N.S. Papageorgiou, Nonlinear Analysis, Chapman \& Hall/CRC, Boca Raton, FL, 2006.

[3] L. Gasinski, N.S. Papageorgiou, Exercises in Analysis, Part 1, Springer, Cham, 2014.

[4] L. Gasinski, N.S. Papageorgiou, Exercises in Analysis, Part 2: Nonlinear Analysis, Springer, Cham, 2016.

[5] L. Gasinski, N.S. Papageorgiou, Positive solutions for nonlinear elliptic problems with dependence on the gradient, J. Differential Equations 263 (2017), 1451-1476.

[6] M. Ghergu, V.D. Rădulescu, Singular Elliptic Problems, Bifurcation and Asymptotic Analysis, Oxford Lecture Series in Mathematics and its Applications, vol. 37, Oxford University Press, Oxford, 2008.

[7] J. Giacomoni, I. Schindler, P. Takač, Sobolev versus Hölder local minimizers and existence of multiple solutions for a singular quasilinear equation, Ann. Sci. Norm. Super. Pisa, Cl. Sci. (5) 6 (2007), 117-158.

[8] M. Girardi, M. Matzeu, Positive and negative solutions of a quasilinear elliptic equation by a mountain pass method and truncature techniques, Nonlinear Anal. 59 (2004), 199-210.

[9] A. Granas, J. Dugundji, Fixed Point Theory, Springer-Verlag, New York, 2003.

[10] E. Hewitt, K. Stromberg, Real and Abstract Analysis, Springer-Verlag, New York, 1975.

[11] N. Hirano, C. Saccon, N. Shioji, Brezis-Nirenberg type theorems and multiplicity of positive solutions for a singular elliptic problem, J. Differential Equations 245 (2008), 1997-2037.

[12] S. Hu, N.S. Papageorgiou, Handbook of Multivalued Analysis. Volume I: Theory, Kluwer Academic Publishers, Dordrecht, The Netherlands, 1997.

[13] N.B. Huy, B.T. Quan, N.H. Knanh, Existence and multiplicity results for generalized logistic equations, Nonlinear Anal. 144 (2016), 77-92.

[14] G. Lieberman, Boundary regularity for solutions of degenerate elliptic equations, Nonlinear Anal. 12 (1988), 1203-1219.

[15] D. Motreanu, V. Motreanu, N.S. Papageorgiou, Topological and Variational Methods with Applications to Nonlinear Boundary Value Problems, Springer, New York, 2014.

[16] N.S. Papageorgiou, V.D. Rădulescu, Combined effects of singular and sublinear nonlinearities in some elliptic problems, Nonlinear Anal. 109 (2014), 236-244.

[17] N.S. Papageorgiou, V.D. Rădulescu, Multiple solutions with precise sign for nonlinear parametric Robin problems, J. Differential Equations 256 (2014), 2449-2479.

[18] N.S. Papageorgiou, V.D. Rădulescu, Nonlinear nonhomogeneous Robin problems with superlinear reaction term, Adv. Nonlinear Studies 16 (2016), 737-764.

[19] N.S. Papageorgiou, V.D. Rădulescu, D.D. Repovš, Pairs of positive solutions for resonant singular equations with the p-Laplacian, Electr. J. Differential Equations 2017, No. 249, pp. 22.

[20] N.S. Papageorgiou, V.D. Rădulescu, D.D. Repovš, Nonlinear elliptic inclusions with unilateral constraint and dependence on the gradient, Appl. Math. Optim. 78 (2018), 1-23.

[21] N.S. Papageorgiou, V.D. Rădulescu, D.D. Repovš, Nonlinear Analysis - Theory and Methods, Springer Monographs in Mathematics. Springer, Cham, 2019.

[22] N.S. Papageorgiou, G. Smyrlis, Nonlinear elliptic equations with singular reaction, Osaka J. Math. 53 (2016), 489-514.

[23] N.S. Papageorgiou, G. Smyrlis, A bifurcation-type theorem for singular nonlinear elliptic equations, Methods Appl. Anal. 22 (2016), 147-170.

[24] K. Perera, Z. Zhang, Multiple positive solutions of singular $p$-Laplacian problems by variational methods, Bound. Value Problems 2009 (2005), 377-382.

[25] P. Pucci, J. Serrin, The Maximum Principle, Progress in Nonlinear Differential Equations and their Applications, vol. 73, Birkhäuser Verlag, Basel, 2007.

[26] D. Ruiz, A priori estimates and existence of positive solutions for strongly nonlinear problems, J. Differential Equations 199 (2004), 96-114.

[27] Y. Sun, S. Wu, Y. Long, Combined effects of singular and superlinear nonlinearities in some singular boundary value problems, J. Differential Equations 176 (2001), 511-531. 
POSITIVE SOLUTIONS FOR NONLINEAR NEUMANN PROBLEMS

(N.S. Papageorgiou) Department of Mathematics, National Technical University, Zografou Campus, 15780 Athens, Greece \& Institute of Mathematics, Physics and Mechanics, Jadranska 19, 1000 Ljubljana, SLOVENIA

(V.D. Rădulescu) Institute of Mathematics, Physics and Mechanics, 1000 Luubluana, Slovenia \& Faculty of Applied Mathematics, Agh University of Science and Technology, 30-059 Kraków, Poland \& Institute of Mathematics "Simion Stollow" of the Romanian Academy, 014700 Bucharest, Romania

(D.D. Repovš) Faculty of Education and Faculty of Mathematics and Physics, University of Ljubluana, 1000 Ljubljana, Slovenia \& Institute of Mathematics, Physics and Mechanics, 1000 Ljubluana, Slovenia

Email address: dusan.repovs@guest.arnes.si 